\title{
Bilateral ureterolithiasis: A true positive and false negative PoCUS exam in a patient with renal colic
}

\author{
Matthew P. Lowe, BSc*; Christopher Cox, MA, MD, DABEM ${ }^{\dagger}$
}

\section{ABSTRACT}

Acute flank pain from suspected urolithiasis is a common presenting complaint in the Emergency Department. Multiple computed tomography (CT) has traditionally been the standard imaging modality used to diagnose obstructive kidney stones, however point of care ultrasound (PoCUS) can play an important role in the diagnostic algorithm and risk stratification of acute flank pain. Here, we present the case of a 29-yearold female with suspected urolithiasis, who underwent PoCUS that revealed right-sided hydronephrosis and normal left kidney, bladder, and aorta. A subsequent KUB was negative. As the clinical course failed to improve with therapy, an abdominal and pelvic CT was ordered revealing a $5 \mathrm{~mm}$ distal obstructing ureteric calculus at the right vesicoureteric junction and another $5 \mathrm{~mm}$ left mid ureteric calculus. To the best of our knowledge, this is the first case in which a patient presenting with acute right-sided flank pain demonstrated unilateral hydronephrosis on PoCUS, but had clinically significant bilateral ureteric stones on CT. Emergency physicians who employ PoCUS for evaluation of flank pain must be aware of its benefits and drawbacks and how they apply to each patient. As such, we have developed a script emergency physicians can use for shared decision-making with renal colic patients when deciding on the appropriate imaging modality.

\section{RÉSUMÉ}

Les douleurs aiguës dans le flanc, vraisemblablement causées par une lithiase urinaire sont un motif fréquent de consultation au service des urgences. Le recours à la tomodensitométrie (TDM) multi-détecteurs comme examen d'imagerie dans la pose du diagnostic de lithiase rénale obstructive est pratique courante, mais l'échographie au point de service (EPS) peut jouer un rôle important dans l'algorithme de diagnostic des douleurs aiguës latérales et de stratification des risques. Sera exposé ici le cas d'une femme de 29 ans, souffrant d'une lithiase urinaire présumée, chez qui l'EPS montrait une hydronéphrose du côté droit mais des images normales de l'aorte, de la vessie et du rein gauche. Un examen ultérieur des reins, de l'uretère et de la vessie a aussi donné des résultats négatifs. Devant l'absence d'une amélioration de l'état clinique malgré le traitement, une TDM du bassin et de l'abdomen a été demandée et a révélé la présence, dans la partie distale de l'uretère, d'un calcul de $5 \mathrm{~mm}$, qui obstruait la jonction vésico-urétérale droite et d'un autre calcul de $5 \mathrm{~mm}$, situé à mi-chemin dans l'uretère gauche. Il s'agit, à notre connaissance, du premier cas dans lequel une hydronéphrose unilatérale a été détectée à l'EPS chez une patiente éprouvant une douleur aiguë du côté droit, mais dans lequel la TDM a révélé la présence bilatérale de calculs dans les uretères, accompagnée d'importants symptômes cliniques. Les médecins d'urgence qui ont recours à l'EPS dans l'évaluation des douleurs dans le flanc doivent connaître les avantages et les inconvénients de l'examen, ainsi que son application dans chacun des cas. Aussi avons-nous élaboré une marche à suivre à l'intention des médecins d'urgence afin de les guider dans les prises de décision par consensus, relatives au traitement des patients atteints de colique néphrétique, quant au type d'examen par imagerie le plus approprié.

Keywords: emergency department, point of care ultrasound, renal, ureteric, kidney stones, shared decision-making

\section{INTRODUCTION}

Acute flank pain from suspected urolithiasis is a common presenting complaint in the emergency department (ED). In addition to renal colic, these patients can present with nausea, vomiting, fever, hematuria, dysuria, and urgency. Computed tomography (CT) has traditionally been the standard imaging modality used to diagnose obstructive kidney stones. However, concerns regarding radiation exposure and time delays, combined with increased training of emergency physicians, have led to the adoption of point-of-care ultrasound (PoCUS) as part of the diagnostic algorithm and risk stratification of acute flank pain. ${ }^{1}$

One such clinical pathway describes a PoCUS-first evaluation for the presence of hydronephrosis, a common secondary sign for obstructive urolithiasis (see Figure $18 \mathrm{~b}$ in Cox et al. for more details). ${ }^{2}$ If hydronephrosis is not seen initially on PoCUS, the patient is given a fluid bolus and the kidneys are rescanned. If hydronephrosis is not visualized but the patient continues to exhibit symptoms suggestive of renal colic, a clinically significant obstruction cannot be ruled out; however, other etiologies should also be considered. If PoCUS is positive for unilateral hydronephrosis, patients are further imaged with a kidneys,

From the *Faculty of Medicine, Dalhousie University, Halifax, NS; and the tDepartment of Emergency Medicine, Dalhousie University, Halifax, NS.

Correspondence to: Christopher Cox, Emergency Medicine, Halifax Infirmary, 355-1796 Summer St., Halifax, NS B3H 3A7, Canada; E-mail: chriscox@dal.ca 
ureters, and bladder (KUB) X-ray. CT imaging and/or a urology consultation is recommended for patients with a stone $>5 \mathrm{~mm}$ identified on KUB X-ray, or any suspected renal stone with persistent pain, elevated creatinine, or clinical signs of infection, regardless of the KUB findings.

\section{CASE}

A previously healthy, 29-year-old female presented to the ED with sudden onset right-sided flank pain beginning 3 hours earlier. The patient described the pain as severe (9/10), sharp, and colicky, with concurrent nausea and vomiting, dysuria and hematuria, but no anuria at any point. A past medical history was significant for previous kidney stones, most recently in 2015 , requiring ureteric stents and lithotripsy. She noted that her symptoms upon presentation were similar but more severe compared to those she had experienced in the past with renal colic. Her medications included quetiapine, clomipramine, and minocycline.

On exam, the patient was afebrile, with a heart rate of 123 beats/min., respiratory rate of 16 breaths/min., and blood pressure of $160 / 118$. Her abdomen was soft with no tenderness or guarding. A genitourinary exam revealed right flank tenderness with no left-sided findings. Respiratory, cardiovascular, and neurologic exams were normal.

In the $\mathrm{ED}$, the patient was given ketorolac $30 \mathrm{mg}$ intravenously (IV), ondansetron $4 \mathrm{mg} I \mathrm{~V}$, and morphine $5 \mathrm{mg}$ IV. The patient was also given $1 \mathrm{~L}$ bolus IV to enhance visualization of hydronephrosis on PoCUS if present.

Bloodwork included a complete blood count (CBC), electrolytes, blood urea nitrogen (BUN), creatinine, and glucose, all of which were normal. Point-of-care Beta hCG was negative. Point-of-care urinalysis was positive for the presence of protein, ketones, bilirubin, and blood.

PoCUS revealed right-sided hydronephrosis (Figure 1). Left kidney, bladder, and aorta were all normal. KUB did not reveal calculi in the renal pelvis, ureter, or bladder, but radiology noted that overlying bowel gas and stool obscured a definitive assessment.

As the clinical course (demonstrated by intractable flank pain) failed to improve over 5 hours with symptomatic therapy and the diagnosis of renal colic yet to be confirmed, an abdominal and pelvic CT was ordered. This study revealed a small 5 -mm distal obstructing ureteric calculus at the right vesicoureteric junction causing proximal moderate hydroureteronephrosis
(Figure 2). Coincidentally, the CT also revealed another 5 -mm left proximal ureteric calculus at approximately the level of the L3 vertebra causing mild ipsilateral proximal hydroureter.

With visualization of the ureteric stones, the etiology of the renal colic was confirmed. The patient's pain was finally adequately controlled, and an urgent follow-up with urology for coordination of an outpatient stent placement was arranged. Urology followed the patient as an outpatient, and 2 weeks later she underwent a bilateral uteroscopy, at which time her right-sided pain had resolved but left-sided pain was now present. The uteroscopy revealed that both the right and left ureteric stones had passed. Four stones in the left renal calices were identified, fragmented with laser lithotripsy, and removed. She then received bilateral ureteric stents.

\section{DISCUSSION}

The incidence of unilateral ureteric obstruction secondary to a stone is around $20 \%$; however, a presentation of bilateral obstructing calculi is uncommon. ${ }^{3-5}$ We have presented, to the best of our knowledge, the first case in which a patient presenting with acute unilateral flank pain demonstrated unilateral nephrolithiasis on PoCUS, but bilateral ureteric stones on CT. Right hydronephrosis on a PoCUS exam correctly predicted the presence of an obstructing stone; however, the absence of left hydronephrosis on PoCUS was a false negative study compared to CT, the gold standard. Fortunately, for our patient, the persistent severity of her symptoms led to subsequent imaging and diagnosis. This avoided the potential pitfall of acute renal failure, a known complication of bilateral obstructive uropathy.

There are a number of factors that may have accounted for our patient's left ureteric calculus not being identified prior to the CT. Firstly, the patient's symptoms, primarily the presence of right-sided flank pain and the absence of pain on the left, clinically indicated unilateral pathology. Distraction from a painful source has been suggested to confound the physical exam in other clinical pathways, such as the NEXUS C-spine injury screening tool, so it is possible this occurred in our case. Secondly, the patient was scanned using PoCUS, a highly operator-dependent tool. ${ }^{6}$ This was unlikely to be an influential factor, however, because the scan was performed by a 
B

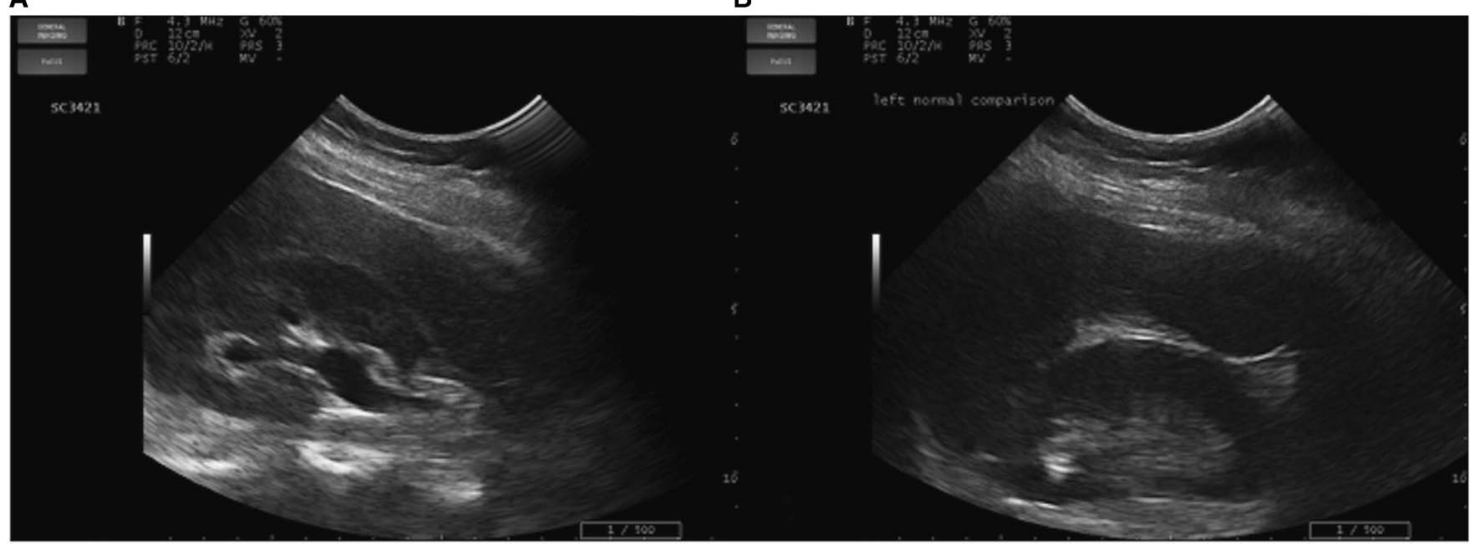

Figure 1. Renal imaging with PoCUS. A) Right kidney showing hydronephrosis. B) Left kidney normal comparison.

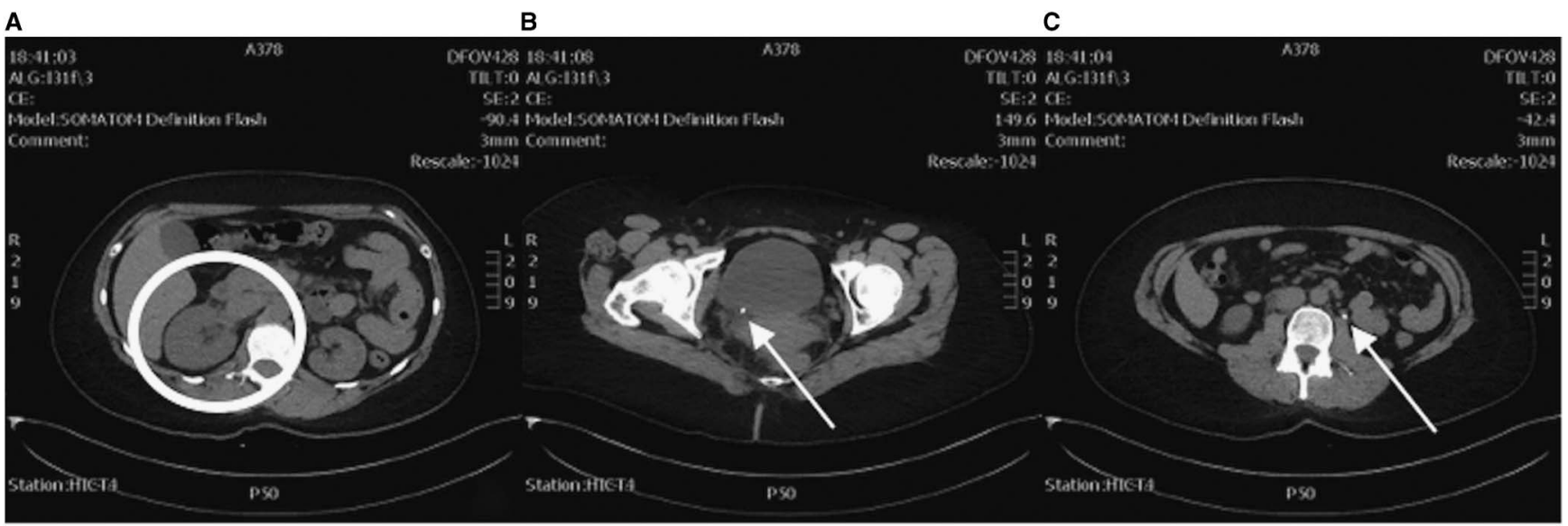

Figure 2. CT axial views. A) Enlarged right kidney indicative of hydronephrosis (circle) compared to the left. B) A 5-mm right distal ureteric calculus at the right vesicoureteric junction (arrow). C) A 5-mm left proximal ureteric calculus (arrow).

physician with CPoCUS Master Instructor credentials and more than 15 years of PoCUS experience. PoCUS is used to visualize hydronephrosis, a potential secondary consequence of obstructing calculi, and not the stones themselves. Ultrasound has a sensitivity and specificity for hydronephrosis ranging from $72 \%$ to $89 \%$ and $73 \%$ to $98 \%$, respectively, but the degree of hydronephrosis can vary based on the size of the stone, hydration status, and the degree of obstruction. ${ }^{1,7,8}$ It is possible that the left ureteric stone created an incomplete obstruction or that insufficient time had elapsed to produce hydronephrosis.

Several studies have examined the role of bedside ultrasound in the diagnosis of renal colic. One study evaluating the validity of PoCUS in Noble and Brown's algorithm for acute flank pain found that more than 50\% of the patients in their study who presented to the ED with acute flank pain were safely discharged following a urinalysis and PoCUS without further investigations. ${ }^{9,10}$ Moak et al. found that the sensitivity and specificity of PoCUS for hydronephrosis resulting from kidney stones were $76.3 \%$ and $59.4 \%$, respectively, and increased to $90.0 \%$ and $63.9 \%$ for stones $>5 \mathrm{~mm}^{11}$ Most recently, Smith-Bindman et al. compared the effectiveness of three renal colic imaging pathways: PoCUS, diagnostic imaging ultrasound, and CT. ${ }^{12}$ The randomized multicentre trial found no inter-group differences in high-risk diagnoses, serious adverse events, pain scores, or hospitalizations, and lower radiation exposure in the ultrasound groups compared to those in the CT pathway. The authors did, however, note that ultrasound-only patients were $40 \%$ more likely to return to the $\mathrm{ED}$ for subsequent imaging.

These studies indicate that a thorough history, urinalysis, and PoCUS may be sufficient for the diagnosis of kidney stones without CT imaging and potential 
exposure to radiation. However, the case that we have presented, along with the sensitivity of the renal PoCUS algorithm, demonstrates that this workup is not always adequate and that further imaging may be warranted. One possibility is diagnostic ultrasound performed by radiology, which is more sensitive than PoCUS. ${ }^{13}$ However, in many centres including ours, a radiology performed ultrasound is only available at 0800 to 1600 hours, so this is not an option for patients presenting outside of this timeframe. KUB X-ray is also reasonable considering its lower radiation dose, $0.7 \mathrm{mSV}$ compared to 10-12 $\mathrm{mSV}$ with CT and 1-3 $\mathrm{mSV}$ with low dose CT. ${ }^{14}$ However, it is less sensitive and may not exclude the need for further imaging, so CT is the best available imaging modality to confirm a diagnosis.

In most cases, the radiation exposure from one CT scan is unlikely to cause significant harm to a patient. For patients with recurrent ureteric stones, however, exposure

Box 1. A script for emergency physicians using shared decision-making with patients with renal colic and confirmed hydronephrosis on ultrasound

Step 1: Acknowledge that a clinical decision needs to be made.

Step 2: Share information in regard to management options and the potential harms, benefits, and outcomes of each.

Step 3: Explore patient values, preferences, and circumstances.

Step 4: Decide together on the best option for the patient, given his or her values, preferences, and circumstances.
"When a patient comes to the emergency department with flank pain, we often consider the possibility of kidney stones. An ultrasound of your kidneys showed something called hydronephrosis, a back-up of fluid in the kidney. We now have a decision to make going forward, so I will explain our options and we can decide on the best course of action together."

"Based on your ultrasound, you likely have a kidney stone blocking one of your ureters. In cases like yours, we commonly do a CT scan to confirm the diagnosis because it is the best way to see kidney stones. A CT is like multiple powerful X-rays, and the radiation from this test can slightly increase your risk of developing cancer. You may also have to wait a few hours to have it performed. The other option is to not get a CT and use the results of your ultrasound. There is some research now showing that urinalysis and ultrasound might be just as effective for diagnosing kidney stones, while avoiding exposure to radiation. The downside is that the ultrasound can miss some stones and some conditions that mimic kidney stones, which may be seen with a CT. So, if your pain got worse or your symptoms did not improve, you would have to come back."

"How do you feel about these options? If you have had kidney stones in the past, have you had any complications and are you worried about complications now? Is it more important for you to avoid exposure to radiation? Do you have time or financial concerns that prevent you from staying for the test today? Would it make you feel better to have the test and determine your diagnosis?"

"Based on your history and symptoms, I can share my recommendations. Since you may be prone to kidney stones, you may need more CTs in the future. Also, most kidney stones pass on their own. So, I would avoid getting a CT today. If you have issues or need to have a procedure in the future, oftentimes you will need another CT anyway, so waiting until then would be better to minimize your exposure to radiation. However, if you would feel more comfortable with a CT scan today, I can arrange that.” 
to radiation must be considered. Our patient had a history of renal colic and had previously undergone three abdominal and pelvic CT scans. Avoiding another CT in this woman of child-bearing age should be preferred. Moreover, a recurrence of ureterolithiasis in patients like ours is common, thus potentially requiring additional CTs in the future. ${ }^{14}$ This is not an insignificant amount of radiation for a disease that does not typically produce complications or require surgical intervention. Newer low dose (1-3 mSV) renal CT protocols have been suggested to lessen the risk to the patient but are currently variable in their availability and radiation dose. In addition, waiting for a CT can result in a time delay to diagnosis, depending on its availability. With all of this in mind, the question of whether a patient with suspected renal stones requires formal imaging is an important opportunity for shared decision-making.

Probst et al. have proposed a framework for shared decision-making in the ED, which they argue is applicable in cases where 1) there is more than one reasonable medical option, 2) the patient is willing and able to make decisions, and 3) there is sufficient time. ${ }^{15}$ This framework can be applied to cases of renal colic. As we have discussed, there is growing evidence for avoiding CT scans in patients with suspected kidney stones by using different imaging pathways. A patient's ability and willingness to engage in shared decision-making can be directly assessed in conversation, and time and patient safety are typically not issues because acute decompensations rarely develop from ureterolithiasis. Therefore, Box 1 outlines a potential script that we have developed based on the four steps of shared decisionmaking in Probst et al.'s study. This discussion compares urinalysis and ultrasound versus additional imaging with CT, the current gold standard. Emergency physicians can use and adapt this script for shared decision-making with patients with renal colic.

\section{CONCLUSION}

Shared decision-making is an important practice in the ED and can be used by emergency physicians using PoCUS algorithms for the evaluation of flank pain. PoCUS is a valuable tool with proven benefits, including reducing radiation exposure and potentially decreasing time to diagnosis, but has lower sensitivity and specificity compared to CT. The case we have presented is the first example in the literature of a patient with unilateral hydronephrosis on PoCUS, but bilateral stones on CT. This report provides cautionary information to clinicians, which should be considered when engaging in shared decision-making discussions with renal colic patients.

Competing interests: None declared.

\section{REFERENCES}

1. Rosen CL, Brown DF, Sagarin MJ, et al. Ultrasonography by emergency physicians in patients with suspected ureteral colic. 7 Emerg Med 1998;16:865-70.

2. Cox C, MacDonald S, Henneberry R, Atkinson PR. My patient has abdominal and flank pain: identifying renal causes. Ultrasound 2015;23(4):232-50.

3. Hou SH, Bushinsky DA, Wish JB, et al. Hospital-acquired renal insufficiency: a prospective study. Am 7 Med 1983;74: 243-8.

4. Vadhwana B, Carey G, Bell DJ. Anuria and acute kidney injury: an uncommon case of bilateral synchronous ureteric calculi. Am 7 Emerg Med 2016;34:2256.e1-2.

5. Stone JR, Knutson TL, Kang C. Bilateral obstructing renal stones: an uncommon cause of acute renal failure. Am $\mathcal{J}$ Emerg Med 2016;28:390.e5-6.

6. Herbst MK, Rosenberg GR, Daniels B, et al. Effect of provider experience on clinician-performed ultrasonography for hydronephrosis in patients with suspected renal colic. Ann Emerg Med 2014;64:269-76.

7. Mandavia DP, Aragona J, Chan L, et al. Ultrasound training for emergency physicians - a prospective study. Acad Emerg Med 2000;7:1008-14.

8. Gaspari R, Horst K. Emergency ultrasound and urinalysis in the evaluation of patients with flank pain. Acad Emerg Med 2005;12:1180-4.

9. Kartal M, Eray O, Erdogru T, Yilmaz S. Prospective validation of a current algorithm including bedside US performed by emergency physicians for patients with acute flank pain suspected for renal colic. 7 Emerg Med 2006;23:341-4.

10. Noble VE, Brown DFM. Renal ultrasound. Emerg Med Clin N Am 2004;22:641-59.

11. Moak JH, Lyons MS, Lindsell CJ. Bedside renal ultrasound in the evaluation of suspected ureterolithiasis. Am 7 Emerg Med 2012;30:218-21.

12. Smith-Bindman R, Aubin C, Bailitz J. Ultrasonography versus computed tomography for suspected nephrolithiasis. N Engl 7 Med 2014;371:1100-10.

13. Metzler IS, Smith-Bindman R, Wang RC, et al. Emergency department imaging modality effect on surgical management of nephrolithiasis: a multicenter, randomized clinical trial. 7 Urol 2017;197:710-4.

14. Gervaise A, Gervaise-Henry C, Pernin M, et al. How to perform low-dose computed tomography for renal colic in clinical practice. Diagn Interv Imaging 2016;97:393-400.

15. Probst MA, Kanzaria HK, Schoenfeld EM, et al. Shared decision-making in the emergency department: a guiding framework for clinicians. Ann Emerg Med 2017;70:688-95. 